\title{
Efeitos do uso de Órteses na Doença de Charcot- Marie-Tooth: Atualização da Literatura
}

\author{
Orthoses Effects in Charcot-Marie-Tooth Disease: Update \\ Rouse Barbosa Pereira', Marco Orsini², Arthur de Sá Ferreira², Júlio Guilherme Silva², Clynton Lourenço \\ Corrêa ${ }^{3}$, Marcos RG De Freitas ${ }^{4}$, Valéria Marques Coelho ${ }^{5}$, Mario Cézar Goulart", Nelson Kagohara6, \\ Gabriela Guerra Leal Souza7, Sara Lúcia de Menezes ${ }^{2,3}$
}

\begin{abstract}
RESUMO I A Doença de Charcot-Marie-Tooth (DCMT) é a neuropatia periférica hereditária mais comum em seres humanos, apresentando incidência de 1:2.500 pessoas. A fraqueza distal crural na DCMT provoca inúmeras alterações na marcha, como, por exemplo, na velocidade, no comprimento, na largura e cadência dos passos. Vários recursos em reabilitação têm sido propostos para gerenciar os problemas de deambulação, dentre eles, destaca-se a utilização de órteses. O objetivo deste estudo é apresentar e discutir os resultados de estudos sobre os efeitos da utilização de órteses nos padrões de marcha na DCMT. Neste estudo foi utilizada atualização da literatura através das principais bases de dados nacionais/internacionais (SciELO, LILACS e MEDLINE), publicados entre os anos de 2006-2012. O tratamento da DCMT consiste em fisioterapia e utilização de equipamentos de assistência, visto que ainda não há fármacos ou terapia gênica capaz de atenuar os danos clínicos e funcionais. Tal associação busca maximizar a função e melhorar a qualidade de vida desses pacientes, na tentativa de evitar agravos adicionais relativos à incapacidade física. A partir de atualização de literatura é possível concluir que existe consenso sobre a utilização de órteses nos membros inferiores para promover a estabilização das articulações do tornozelo e um padrão de deambulação mais funcional, evitando sinergias inadequadas de movimento e atenuando o risco de quedas. Descritores I Doença de Charcot-Marie-Tooth; Aparelhos Ortopédicos; Reabilitação.
\end{abstract}

\begin{abstract}
I Charcot-Marie-Tooth (CMT) disease is the most common hereditary peripheral neuropathy in humans, presenting incidence of 1:2.500 people. The distal crural weakness of the CMT causes numerous gait impairment changes, for example, velocity, length, width and cadence of the steps. Several rehabilitation resources have been proposed to manage the walking problems, among them, the use of orthoses is highlighted. The objective of this study was to present and discuss the results of studies on the effects of orthotic uses in CMT gait patterns. In this study it was used to literature update through major national/international databases (SciELO, LILACS and MEDLINE), published between the years 2006-2012. The CMT treatment consisted of physical therapy and using of assistive equipment, since there are no drugs or gene therapy able to attenuate the clinical and functional damages. This association seeks to maximize the function and improve life quality of these patients in an attempt to prevent additional injuries related to physical disability. From literature update it is possible to conclude that there is a consensus on the use of lower limb orthoses to promote the stabilization of the ankle articulation and a more functional pattern of walking, avoiding inadequate synergy of movement and reducing the risk of falls.
\end{abstract}

Keywords I Charcot-Marie-Tooth disease; Orthotic Devices; Rehabilitation.

Estudo desenvolvido no Centro Universitário Augusto Motta (UNISUAM), Programa de Mestrado em Ciências da Reabilitação Bonsucesso (RJ), Brasil.

'Fisioterapeuta; Mestranda no Programa de Pós-Graduação em Ciências da Reabilitação (PPGCR) pelo UNISUAM - Bonsucesso (RJ), Brasil. ${ }^{2}$ Professores Doutores do PPGCR pelo UNISUAM - Bonsucesso (RJ), Brasil.

${ }^{3}$ Professores Doutores do Curso de Fisioterapia da Universidade Federal do Rio de Janeiro (UFRJ) - Rio de Janeiro (RJ), Brasil.

${ }^{4}$ Professor Titular e Chefe do Serviço de Neurologia da Universidade Federal Fluminense (UFF) - Niterói (RJ), Brasil.

${ }^{5}$ Fisioterapeutas da Associação Fluminense de Reabilitação (AFR) - Niterói (RJ), Brasil.

${ }^{6}$ Médico Fisiatra da AFR - Niterói (RJ), Brasil.

${ }^{7}$ Fisioterapeuta; Professora Doutora do Departamento de Ciências Biológicas da Universidade Federal de Ouro Preto (UFOP) - Ouro Preto (MG), Brasil. 


\section{INTRODUÇÃO}

A Doença de Charcot-Marie-Tooth (DCMT) é a condição neurológica herdada mais diagnosticada no mundo, com incidência de aproximadamente 1 a cada 2.500 indivíduos. Possui etiologia desconhecida, com características genéticas transmitidas de forma autossômica dominante, recessiva ou recessiva ligada ao X. O gene anormal localiza-se no cromossomo 17 e o distúrbio resulta de uma duplicação de parte dele. Pode ser classificada de acordo com o seu modo de transmissão ou segundo a parte do nervo que é afetada (bainha de mielina ou axônio) ${ }^{1-3}$.

Pacientes com DCMT caracterizam-se clinicamente por evolução lenta e progressiva associada à fraqueza muscular distal crural, principalmente dos músculos intrínsecos do pé, originando pé cavo e dedos em garra, além de déficit muscular da região fibular, provocando o derreamento do pé e a marcha 'escarvante' (Figura 1) 4,5 . As alterações sensitivas podem estar presentes e, quando ocorrem, costumam ser marcadas por: câimbras, parestesias, disestesias, hipoestesia superficial e profunda distal, além de arreflexia ou hiporreflexia tendínea ${ }^{6,7}$.

A avaliação dos padrões da marcha na DCMT é de fundamental importância, pois permite aos profissionais envolvidos com a reabilitação física, o estabelecimento de metas de acordo com as alterações biomecânicas encontradas $^{6,8}$. Visto que atualmente ainda não há fármacos ou forma de terapia gênica para DCMT, o tratamento preconiza a reabilitação funcional e melhora na qualidade de vida desses indivíduos. A reabilitação envolve todas as formas de intervenções terapêuticas, desde as formas conservadoras com a prescrição de fisioterapia e órteses, como as intervenções cirúrgicas para as deformidades mais graves. O objetivo do tratamento é maximizar a função e a manutenção do quadro, proporcionando uma qualidade de vida mais adequada para essa população, na tentativa de evitar uma incapacidade total ${ }^{9,10}$.

As órteses, uma das modalidades terapêuticas empregadas, podem ser classificadas como estáticas, quando imobilizam, limitam e posicionam a articulação; ou como dinâmicas, quando permitem a movimentação articular, promovem neutralização de forças deformantes, manutenção e fortalecimento da musculatura envolvida. Atualmente, vários modelos de órteses têm sido desenvolvidos com diferentes características mecânicas e usando avançadas tecnologias de fabricação, como silicone, carbono, polipropileno e material elástico. Os tipos de órteses podem variar de acordo com a necessidade de cada paciente, podendo ser divididas em: órtese tornozelo-pé (OTP), órtese joelho-tornozelo-pé, órtese quadril-joelho-tornozelo-pé e órtese para membros superiores. Em pacientes com DCMT, o tipo de órtese a ser empregado deve ser avaliado, após criterioso diagnóstico cinético-funcional, seguindo as particularidades da doença e do paciente ${ }^{11,12}$.
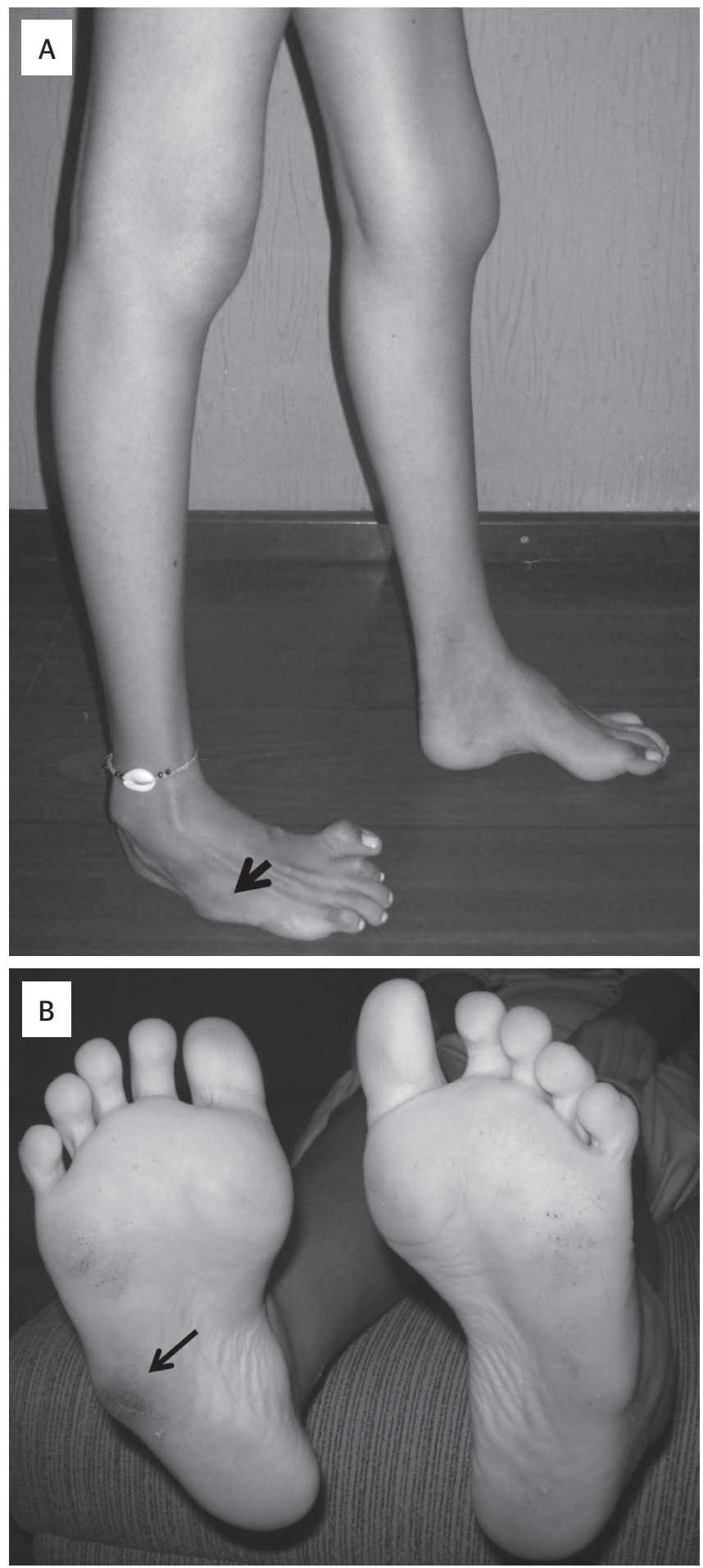

Figura 1. Paciente com DCMT. (A) Paciente com instabilidade articular em tornozelo. Note os dedos fletidos (cabeça de seta) e aumento do arco plantar. (B) Nota-se a alteração do ponto de pressão que favorece deformidade nos pés. 
O objetivo do presente estudo é apresentar e discutir, com base na literatura vigente, os resultados dos principais estudos envolvendo pacientes com DCMT e uso de órteses.

\section{METODOLOGIA}

Foi feita uma atualização da literatura baseada em artigos científicos publicados no período de 2006 a 2012 a respeito do assunto supracitado, através de análise descritiva. Artigos de anos anteriores considerados importantes para fundamentação do arcabouço teórico-conceitual também foram utilizados. Os artigos científicos foram obtidos a partir de pesquisa nas bases de dados Literatura Latino-Americana e do Caribe e Ciências da Saúde (LILACS), Medical Literature Analysis and Retrieval System Online (MEDLINE) e Scientific Electronic Library (SciELO) com os seguintes descritores: Doença de Charcot-Marie-Tooth, Órteses, Equipamentos de assistência, Fisioterapia e Reabilitação e seus equivalentes em inglês, francês e espanhol. A busca eletrônica foi realizada entre janeiro e março de 2012.

Vinte artigos foram encontrados, dentre os quais 14 foram selecionados e analisados, sendo 10 para a fundamentação teórica e 6 para a análise descritiva. Todos os artigos estavam relacionados à DCMT ou órteses, no entanto somente 6 referiam-se especificamente ao uso de órteses em DCMT (Tabela 1). Os artigos que não apresentavam clareza quanto aos métodos de avaliação e/ou intervenções utilizadas e os resultados obtidos, assim como os estudos que eram conduzidos em animais de experimentação, foram excluídos.

\section{RESULTADOS}

Um estudo científico teve como objetivo caracterizar as possíveis diferenças na apresentação clínica e na função da marcha entre pacientes com DCMT que faziam uso regular de OTP (grupo OTP) com aqueles que não as utilizavam (grupo não OTP) ${ }^{13}$. Tais autores mensuraram os parâmetros da função da marcha por meio do teste de tempo de caminhada de $10 \mathrm{~m}$ e do teste de caminhada de 6 min. Foram utilizadas as escalas de Borg e de Avaliação para Charcot-Marie-Tooth (CMTES) para mensurar o esforço percebido durante os testes e para avaliar a gravidade da doença e o déficit sensorial, respectivamente. Em adição, a escala de impacto de caminhada $12 \mathrm{~m}$ foi aplicada para mensurar a percepção dos sujeitos na execução dos padrões e na habilidade de deambulação. Verificaram que não houve diferença significativa na velocidade média normal da marcha durante os testes de $10 \mathrm{~m}(1,07 \pm 0,26$ $\mathrm{m} / \mathrm{s}$ grupo OTP, $1,21 \pm 0,17 \mathrm{~m} / \mathrm{s}$ não OTP) e $6 \mathrm{~min}$

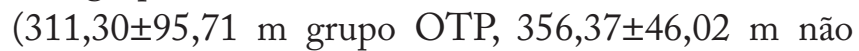
OTP). Em contrapartida, foram encontradas diferenças na velocidade máxima ao longo do teste de $10 \mathrm{~m}$

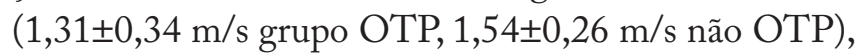
no esforço percebido durante a caminhada no teste de $6 \mathrm{~min}(135,62 \pm 51,84 \mathrm{~m}$ grupo OTP, 105,00 $\pm 15,26 \mathrm{~m}$ não OTP) e na percepção da capacidade de deambular (grupo OTP 43 e não OTP 30). Os resultados mostraram que os usuários de OTP deambulavam de forma mais lentificada e com maior esforço percebido durante a caminhada. Os autores justificaram tais resultados pela maior gravidade da doença no grupo OTP, levando a uma pior percepção na capacidade na execução dos padrões da marcha. Comumente, pacientes que usam OTP tendem a ter uma apresentação clínica mais grave da doença e, apesar do auxílio fornecido por esses dispositivos, não deambulam de forma tão funcional quando comparados com o grupo não OTP.

Em outra proposta de estudo científico ${ }^{14}$, os pesquisadores demonstraram que órteses com diferentes características mecânicas contribuem para melhorar o controle postural e a marcha em pacientes com DCMT. Os autores avaliaram três condições experimentais em

Tabela 1. Estudos sobre o uso de órteses tornozelo-pé em Charcot-Marie-Tooth

\begin{tabular}{|c|c|c|c|}
\hline Autores (data) & Tipos de pesquisa & $\mathrm{n}$ & Principais resultados \\
\hline Guzian et al. (2006) $)^{18}$ & Quantitativa e qualitativa & 1 & SO é excelente meio de tratamento para as alterações da marcha \\
\hline Vinci, Gargiulo (2008)17 & Qualitativa & 25 & A prescrição de OTP deve ser acompanhada com suporte psicológico \\
\hline Del Bianco, Fatone (2008)16 & Quantitativa & 1 & OTP-FMP promoveu melhor controle durante a fase de transição de apoio \\
\hline Vinci et al. $(2010)^{15}$ & Quantitativa & 1 & SMP melhora os padrões da marcha \\
\hline Guillebastre et al. $(2011)^{14}$ & Quantitativa & 26 & A prescrição de OTP parece ser relevante para a melhora do equilibrio e da marcha \\
\hline Ramdharry et al. $(2012)^{13}$ & Quantitativa & 32 & As pessoas com CMT usam órteses quando sua condição torna-se mais grave \\
\hline
\end{tabular}

SO: sapatos ortopédicos; OTP: órtese tornozelo-pé; OTP-FMP: órtese tornozelo-pé com feixe de molas posteriores; SMP: suporte macio para pé caido (soft footdrop insert); CMT: Charcot-Marie-Tooth 
26 pacientes com DCMT, onde esses usavam calçados comuns (CC), calçados associados com OTP de plástico (OTP-P) e calçados associados com OTP de material elástico (OTP-E). O objetivo do estudo foi identificar alterações na força muscular e nas modalidades sensitivas e, a partir dos achados clínicos, selecionar o modelo de OTP mais apropriado para esses pacientes. A força muscular dos flexores plantares e dorsiflexores do tornozelo, flexores e extensores do joelho e flexor e extensor do hálux foram testadas por meio da escala desenvolvida pelo Medical Research Council (MRC). Para a avaliação da postura ereta, utilizou-se uma plataforma de força (PF02, Equi ${ }^{+}$, France). A análise da marcha foi realizada usando um tapete eletrônico (Gaitrite ${ }^{\circledR}$ ) e avaliada a velocidade da marcha, tempo do passo, comprimento do passo e o contato inicial. Os resultados mostraram que o uso de OTP-P ou OTP-E associados com sapatos comuns melhora o controle postural e a marcha, baseado na diminuição significativa na área do centro de pressão e pelo aumento do comprimento do passo quando os pacientes usavam OTP-P ou OTP-E quando comparado somente com CC. Os resultados indicaram também que a OTP-P é mais eficaz para pacientes com déficits musculares mais graves enquanto a OTP-E é mais apropriada para pacientes com força muscular relativamente preservada na parte distal crural. Os pacientes quando em uso da OTP-P obtiveram um melhor desempenho da marcha (houve aumento da velocidade da marcha e no comprimento do passo). Em pacientes capazes de realizar movimento de flexão plantar contra resistência, o uso da OTP-E foi mais apropriado, aumentando a velocidade da marcha.

Com o objetivo de analisar a cinética e a cinemática da marcha de um paciente com DCMT apresentando envolvimento dos músculos proximais e distais dos membros inferiores, pesquisadore ${ }^{15}$ descreveram o uso de três tipos de órteses. Órtese tornozelo-pé de silicone (OTP-S), calçado ortopédico com suporte Codivilla, e um novo dispositivo projetado exclusivamente para esse paciente, o suporte macio para pé-caído (SMP). Esse equipamento de assistência trata-se de uma tala interna localizada na parte posterior da perna e na face plantar (soft footdrop insert). As órteses supracitadas foram usadas por três anos. A análise da marcha com as OTP-S não foi realizada até o término do estudo, por causa do quadro álgico relatado pelo paciente. Em vista disso, o paciente usou seus sapatos comuns no lugar das OTP-S para realizar o estudo. $\mathrm{O}$ uso do SMP forneceu bons resultados durante a análise instrumental da marcha, caracterizados pelos seguintes marcadores: (a) a articulação do tornozelo exibiu dorsiflexão leve quando em contato com o solo, seguido por plantiflexão. Os resultados, na fase de balanço da marcha, foram mais expressivos com a utilização do calçado ortopédico com suporte Codivilla e com o SMP, quando comparados aos sapatos comuns; (b) a articulação do quadril apresentou um aumento na flexão durante a fase de apoio médio. Nessa, os aumentos foram mais marcantes com o uso dos sapatos comuns, intermediários com o SMP e menos marcantes com o suporte Codivilla. As seguintes variáveis foram avaliadas durante a marcha: velocidade média, velocidade de balanço, cadência, duração da fase de postura, comprimento das passadas direita e esquerda, largura dos passos direito e esquerdo. Todos os dados foram coletados no mesmo dia com o valor médio de três tentativas para cada condição. Ao final da análise, o novo dispositivo, SMP, provou ser mais satisfatório para o paciente por não gerar dor e/ou compressão, além de ser mais agradável esteticamente. $\mathrm{O}$ paciente também apresentou melhorias na execução dos padrões da marcha, com realização de menos sinergias inadequadas de movimentos. Vale ressaltar que a aderência de pacientes com DCMT ao uso de OTP ainda é baixa, em vista disso, os autores esperam que o SMP possa representar um ponto de partida na concepção e produção de novos dispositivos ortopédicos mais confortáveis e com maior aceitação estética, para satisfazer tal população.

As funções de um modelo pré-fabricado de órtese tornozelo-pé constituída por silicone (OTP-S) e um outro modelo composto por feixes de molas posteriores (OTP-FMP) foram comparadas, assim como seus efeitos na cinemática e cinética da marcha de um indivíduo com $\mathrm{DMCT}^{16}$. Os autores relataram que ambos os equipamentos de suporte melhoraram os padrões de marcha, quando comparados à condição de usar apenas o calçado. Vale ressaltar que os melhores resultados obtidos ao final do estudo para plantiflexão e dorsiflexão ocorreram com o uso da OTP-FMP, mesmo esta provocando maior resistência na amplitude de movimento do tornozelo. A OTP-FMP também foi capaz de corrigir desvios da marcha (cadência, velocidade, comprimento da passada) nas fases de apoio e balanço, enquanto a OTP-S agiu predominantemente na fase de balanço. O uso da OTP-FMP também foi capaz de criar um momento de dorsiflexão quase normal durante a resposta à carga, enquanto que a OTP-S não apresentou nenhum efeito nessa fase. Os resultados evidenciaram um melhor controle durante a transição de apoio do membro inferior direito para o esquerdo com o uso da OTP-FMP, quando comparado com a deambulação sem órtese. 
A aderência de 25 pacientes com DCMT ao uso de OTP foi avaliada por pesquisadores ${ }^{17}$ a fim de investigar os problemas ortopédicos e psicológicos relacionados com o pé caído grave. A análise foi realizada em um único dia por meio de duas avaliações. A primeira foi realizada por um fisiatra, na qual o paciente respondia se utilizava ou não algum tipo de OTP. Em seguida, uma fotografia dos membros inferiores era registrada com o paciente em posição ortostática usando seus próprios calçados (para aqueles que não faziam uso de OTP) ou a OTP. Por meio dos registros fotográficos, os autores mensuraram o ângulo entre o eixo da perna e a sola do pé para avaliar a presença de pé-caído nesses pacientes com o uso de um goniômetro. A segunda avaliação foi realizada por um psicólogo, que investigou fatores de comprometimento da qualidade de vida, através de entrevista qualitativa, com o objetivo de analisar a relação do paciente com seu próprio corpo e com o uso do dispositivo ortopédico. Essa avaliação demonstrou que todos os pacientes que faziam uso de OTP tinham um mau relacionamento com seu próprio corpo. Esse estudo indicou que a frequência do uso de OTP em pacientes com DCMT apresentando pé-caído grave é muito baixa devido principalmente aos desconfortos físicos gerados (dor e/ou compressão), além de desconforto psicológico. Os autores sugerem que a prescrição de OTP seja acompanhada de suporte psicológico e que futuras pesquisas visando mais conforto e soluções estéticas mais aceitáveis pelos pacientes sejam estimuladas, buscando facilitar a aderência da OTP em pacientes com DCMT.

$\mathrm{O}$ caso de um paciente com DCMT foi descrito; ele apresentava distúrbios na marcha e foi tratado com fisioterapia e utilização de sapatos ortopédicos $(\mathrm{SO})^{18}$. Os $\mathrm{SO}$ foram prescritos visando melhoras na marcha e no equilíbrio do paciente, enquanto a fisioterapia foi prescrita para manter a amplitude de movimento do tornozelo. Os autores realizaram a avaliação da eficácia do tratamento a nível clínico e quantificaram os parâmetros espaciais e temporais da marcha do paciente antes e depois do tratamento. A avaliação quantitativa foi realizada com um sistema de medidas Gaitrite ${ }^{\circledR}$ usado para registrar os parâmetros espaciais e temporais da marcha (comprimento e largura do passo, ângulo entre os pés e a linha de progressão, tempo do passo, ciclo da marcha, velocidade, cadência, tempo de atitude e tempo de rotação). Uma câmera de vídeo foi usada para realizar a análise da marcha através de imagens mostrando o paciente andando no plano sagital e frontal. A primeira avaliação clínica e análise da marcha, realizadas antes do início do tratamento com os $\mathrm{SO}$, tiveram como objetivo registrar a história da doença, as principais queixas do paciente (dor, quedas frequentes, dificuldade em subir e descer escadas e andar em terrenos irregulares) e identificar as deficiências neurológicas e musculoesqueléticas existentes, tais como, pé caído, dedos em garra e déficit de equilíbrio e de sensibilidade. $O$ exame físico e a análise da marcha foram realizados com o paciente descalço e mostraram a existência de distúrbios de equilíbrio envolvendo movimentos compensatórios de balanço e abdução dos membros superiores e um alargamento da base de apoio para os pés. $\mathrm{O}$ paciente ainda apresentou dificuldades de subir e descer escadas com os sapatos normais. A segunda avaliação, realizada após um mês de uso dos SO pelo paciente, visou analisar a eficácia do tratamento e a tolerância do paciente em relação ao SO. O paciente foi questionado sobre os sintomas que sentia antes do tratamento (dor, instabilidade ao andar, quedas, dificuldade de subir e descer escadas) e relatou que esses desapareceram quando começou a usar os $\mathrm{SO}$. Os resultados da análise da marcha mostraram que a estabilidade do tornozelo e o equilíbrio melhoraram; o equino-varo do pé e os movimentos compensatórios dos membros superiores diminuíram; a velocidade da marcha (descalço: $0,38 \mathrm{~m} / \mathrm{s}, \mathrm{SO}: 0,54 \mathrm{~m} / \mathrm{s}$, valor normal: $0,8-1,0 \mathrm{~m} / \mathrm{s}$ ), a cadência (descalço: $66 \mathrm{p} / \mathrm{min}, \mathrm{SO}$ : $74 \mathrm{p} / \mathrm{min}$, valor normal: $75 \mathrm{p} / \mathrm{min}$ ) e o comprimento do passo (direito: descalço: $34,6 \mathrm{~cm}$, SO: $43,1 \mathrm{~cm}$; e esquerdo: descalço: $37,6 \mathrm{~cm}$, SO: $43,8 \mathrm{~cm}$, valor normal: $75 \mathrm{~cm}$ ) aumentaram; houve uma diminuição da área da base de apoio e da largura do passo (direito: descalço: $14,3 \mathrm{~cm}$, SO: $12,4 \mathrm{~cm}$; e esquerdo: descalço: $13,7 \mathrm{~cm}$, SO: $12,1 \mathrm{~cm}$, valor normal: $10-12 \mathrm{~cm}$ ). Os resultados foram satisfatórios, mostrando que tanto os dados clínicos qualitativos, quanto os quantitativos, confirmam a melhora subjetiva percebida pelo paciente. Os autores concluíram que os sapatos ortopédicos são um excelente meio de tratamento para as alterações da marcha em pacientes com DCMT, porém não alcançam os valores normais quando comparados a de um sujeito saudável.

\section{CONCLUSÃO}

A presença de derreamento do pé em doenças neuromusculares provoca inúmeras alterações funcionais. A prescrição de órteses tornozelo-pé em pacientes com DCMT parece relevante, devido a promover melhorias nas reações de equilíbrio e no desempenho da marcha. A realização de tratamento através do uso 
de equipamentos de assistência pode minimizar as sinergias inadequadas de movimento e otimizar a função nesses pacientes.

O pequeno número de trabalhos realizados envolvendo o uso de órteses em pacientes com DCMT, apesar de esta condição neurológica ser uma das mais diagnosticadas do mundo, e o fato de que a maioria dos trabalhos utilizou relato de caso, nos faz sugerir fortemente a realização de mais pesquisas quantitativas nessa área.

\section{REFERÊNCIAS}

1. Fávero RA, Polese JC, De Oliveira SG, Schuster RC. Análise da marcha e funcionalidade na doença de Charcot-Marie-Tooth: relato de caso. Rev Neurociênc. (Impr.). 2010;18(1):44-9.

2. Costacurta MLG, Sousa PPC, Zuccon A, Morais Filho MC, Rocco FM, Gaspar AP. Impacto da correção cirúrgica dos membros inferiores na qualidade de vida de pacientes com a doença de Charcot-MarieTooth. Acta Fisiátrica. 2011:18(1):1-5.

3. Berciano J, Sevilla T, Casasnovas C, Sivera R, Vilchez JJ, Infante J, et al. Guidelines for molecular diagnosis of Charcot-Marie-Tooth disease. Neurología (Barc., Spain). 2012;27(3):169-78.

4. Meningroni PC, Nakada CS, Hata L, Fuzaro AC, Marques Júnior W, Araújo JE. Irradiação contralateral de força para ativação do músculo tibial anterior em portadores da doença de Charcot-Marie-Tooth: efeitos de um programa de intervenção por PNF. Rev Bras Fisioter. 2009;13(5):438-43

5. Don R, Serrao M, Vinci P, Ranavolo A, Cacchio A, loppolo F, et al. Foot drop and plantar flexion failure determine different gait strategies in Charcot-Marie-Tooth patients. Clin Biomech. 2007;22(8):905-16.

6. Maranho DAC, Volpon JB. Pé cavo adquirido na doença de CharcotMarie-Tooth. Rev Bras Ortop. 2009:44(6):479-86.

7. Vieira THF, Aires RD, Mendonça VA, Corrêa CL. Reabilitação física em um paciente com a doença de Charcot-Marie-Tooth: relato de caso.
Rev Neurociênc (Impr.) [periodico on-line] 2008; [cited 2012 Apr 09]. Available from: http://saude.br/dneuro/neurociencias/300_relato.pdf

8. Newman CJ, Walsh M, O'Sullivan R, Jenkinson A, Bennett D, Lynch B, et al. The characteristics of gait in Charcot-Marie-Tooth disease types I and II. Gait Posture. 2007;26(1):120-7.

9. Ferrarin M, Bovi G, Rabuffetti M, Mazzoleni P, Montesano A, Moroni I, et al. Reliability of instrumented movement analysis as outcome measure in Charcot-Marie-Tooth disease: results from a multitask locomotor protocol. Gait Posture. 2011;34(1):36-43.

10. Maldaner M, Oliveira SW, Sachetti A, Schiavinato JCC, Gemelli SO. Fisioterapia aquática na doença de Charcot-Marie-Tooth. Rev Ciênc Méd Biol. 2011;10(2):121-5.

11. Carbonero FC, Zago GM, Campos D. Tecnologia assistiva na Distrofia Muscular de Duchenne: aplicabilidade e benefícios. Rev Neurociênc (Impr.) [periodico on-line] 2011; [cited 2012 Apr 21]. Available from: http:// revistaneurociencias.com.br/inpress/654\%20rev\%2Oinpress.pdf

12. Schrank ES, Stanhope SJ. Dimensional accuracy of ankle-foot orthoses constructed by rapid customization and manufacturing framework. J Rehabil Res Dev. 2011;48(1):31-42.

13. Ramdharry GM, Pollard AJ, Marsden JF, Reilly MM. Comparing gait performance of people with Charcot-Marie-Tooth disease who do and do not wear ankle foot orthoses. Physiother Res Int. 2012; DOl:10.1002/pri.531.

14. Guillebastre B, Calmels P, Rougier PR. Assessment of appropriate ankle-foot orthoses models for patients with Charcot-Marie-Tooth disease. Am J Phys Med Rehabilit. 2011;90(8):619-27.

15. Vinci P, Paoloni M, Ioppolo F, Gargiulo P, Santilli V. Gait analysis in patient with severe Charcot-Marie-Tooth disease: a case study with a new orthotic device for footdrop. Eur J Phys Rehabil Med. 2010;46(3):355-61

16. Del Bianco J, Fatone S. Comparison of silicone and posterior leaf spring ankle-foot orthoses in a subject with Charcot-Marie-Tooth disorder. J Prosthet Orthot. 2008;20(4):155-62.

17. Vinci P, Gargiulo P. Poor compliance with ankle-foot-orthoses in Charcot-Marie-Totth disease. Eur J Phys Rehabil Med. 2008;44(1):27-31.

18. Guzian MC, Bensoussan L, Viton JM, De Bovis VM, Ramon J, Azulay JP, et al. Orthopaedic shoes improve gait in a Charcot-Marie-Tooth patient: a combined clinical and quantified case study. Prosthet Orthot Int. 2006;30(1):87-96 